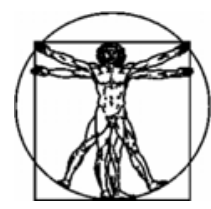

\title{
ОПИСАНИЕ МЫШЕЧНЫХ И СУСТАВНЫХ СИЛ ПРИ НАГРУЖЕНИИ НИЖНЕЙ ЧЕЛЮСТИ. ЭКСПЕРИМЕНТАЛЬНЫЙ И ВЫЧИСЛИТЕЛЬНЫЙ МЕТОДЫ
}

\author{
М. Менар ${ }^{1}$, О. Менар ${ }^{1}$, А. Рамос ${ }^{2}$, В.А. Лохов ${ }^{3}$, Ю.И. Няшин ${ }^{3}$ \\ ${ }^{1}$ Université de Bordeaux, CNRS UMR 5295, Institut de Mécanique et d'Ingénierie, 351 cours de la Libération, \\ 33405 Talence, France, e-mail: michel.mesnard@u-bordeaux.fr \\ Университет Бордо, Франция \\ 2 Biomechanics Research Group, University of Aveiro, 3810-193 Aveiro, Portugal \\ Университет Авейру, Португалия \\ ${ }^{3}$ Кафедра теоретической механики и биомеханики Пермского национального исследовательского \\ политехнического университета, Россия, 614990, Пермь, Комсомольский проспект, 29
}

\begin{abstract}
Аннотация. Представлен трехмерный метод определения численных величин и анализ мышечных сил и усилий в суставах при нагружении нижней челюсти. Экспериментальный протокол, гипотезы, запись и затем решение системы уравнений для статического равновесия нижней челюсти описаны и использованы с помощью данных, полученных у четырех добровольцев. Был сконструирован датчик для моделирования и регистрации силы кусания между двумя зубами, затем он был применен последовательно для двух резцов, двух премоляров и затем двух моляров. Рассечения были проведены для определения контактов между мыщелками нижней челюсти и височной костью, а также для определения мест прикрепления мышц, закрывающих челюсти. Направления мышечных сил были затем определены в морфологической системе координат. Магнитно-резонансная диагностика и электромиография были далее применены in vivo для оценки величин шести мышечных сил на каждой стороне лица. Решение системы уравнений, полученной для вычисления сил, передаваемых через суставные контакты, было проведено с помощью компьютера. Для идентичного нагружения, примененного последовательно в положениях 31, 34 и 36, на основе 12 показаний результаты показывают, что сагиттальные компоненты суставных сил сильно зависят от межзубного расстояния. В положении 31 вертикальная компонента умножается на 1,65, когда межрезцовое расстояние увеличивается от 5 до 15 миллиметров. Величины суставных сил сильно зависят от величины открытия рта и от направлений результирующих мышечных сил.
\end{abstract}

Ключевые слова: биомеханическое описание, мышечные силы, суставные силы, механическое моделирование, имплантат височно-нижнечелюстного сустава.

\section{ВВеДЕНИЕ}

Реконструкция височно-нижнечелюстного сустава была проведена, чтобы улучшить функцию нижней челюсти и уменьшить нарушение трудоспособности.

(C) Менар М., Менар А., Рамос А., Лохов В.А., Няшин Ю.И., 2015

Менар Мишель, профессор, институт механики и инженерии и отделение зубочелюстной ортопедии, Бордо

Менар Оде, студентка кафедры науки о жизни, Бордо

Рамос Антонио, ассистент профессора кафедры механической инженерии, Авейру

Лохов Валерий Александрович, к.ф.-м.н., доцент, заведующий кафедрой теоретической механики и биомеханики, Пермь

Няшин Юрий Иванович, д.т.н., профессор, научный руководитель кафедры теоретической механики и биомеханики, Пермь 
Некоторые протезы височно-нижнечелюстного сустава не нашли широкого применения. Хроническая боль или воспаление указывало на микроперемещения имплантата $[1,8,11]$. Связь с костью была подвержена разрушению, если величины нагрузки не были должным образом учтены $[17,19]$. Эти проблемы, включающие знание нагрузок, деформаций и/или напряжений, требуют дополнительных данных, характеризующих биомеханику естественного сустава.

Конструкция протеза сустава должна учесть естественные биомеханические данные. Чтобы охарактеризовать здоровый височно-нижнечелюстной сустав, приближение должно быть к кинематике (геометрия, перемещения) и квазистатике (силы, деформации, напряжения), которые при этом дополняют друг друга. Экспериментальный протокол, основанный на анализе функционирования, был получен Менаром [12]. Этот результат обусловил направление наших исследований и выбор экспериментальных методов. Кинематика нижней челюсти и перемещения в суставе были определены с использованием $3 D$-видеоанализа [5], чтобы оценить мышечные и суставные силы, действующие на нижнюю челюсть.

Throckmorton показал, что относительные величины сил в мышцах челюсти определяют направление сил в височно-нижнечелюстном суставе [18]. Он пришел к выводу, что результаты вычислений менее чувствительны к ошибкам, когда число мышц, взятых в расчет, увеличивается.

Koolstra показал, что разность в поперечных сечениях мышц челюсти слабо зависит от величины силы кусания [10]. Он также показал, что результаты биомеханических вычислений сил в височно-нижнечелюстном суставе сильно зависят от изменений направления сил.

\section{МАТЕРИАЛЫ И МЕТОДЫ}

Этот раздел описывает биомеханическую модель, разработанную для измерения сил в мышцах при закрытии челюсти и оценки нагрузок в височно-нижнечелюстных суставах при статическом равновесии нижней челюсти при межзубном нагружении.

\section{Биомеханическая модель и волонтеры}

Было предположено, что лицо имеет морфологическую симметрию относительно сагиттальной плоскости.

Отсчетная система координат

В большинстве статей, где анализируются движения челюсти или воздействия на нижнюю челюсть, для предоставления результатов используется неопределенная или экзотическая система координат. В данном исследовании используется функциональная и анатомическая система координат (рис. 1). Она $\left(S_{c}\right)$ связана с тремя морфологическими точками, определяющими плоскость Кампера: точка $N$ - угловая точка соединения носовой перегородки и верхней губы и точки $L$ и $R$ - центры левого и правого мыщелков в положении бугорково-фиссурного контакта зубов-антагонистов. Определение системы координат $S_{c}$ имеет следующий вид:

- Начало $O_{c}$ есть середина сегмента, определенного двумя центрами мыщелков.

- Ось $x$ проходит через центры двух височно-нижнечелюстных суставов (положительное направление оси $x$ - влево).

- Ось $z$ определяется точками $O_{c}$ и $N$ (положительное направление оси $z$ от точки $O_{c}$ к точке $N)$.

- Ось $y$ заканчивает систему (перпендикуляр к плоскости Кампера, положительное направление оси $y$ - вверх). Базис, связанный с этими тремя осями, обозначается как $b$. 


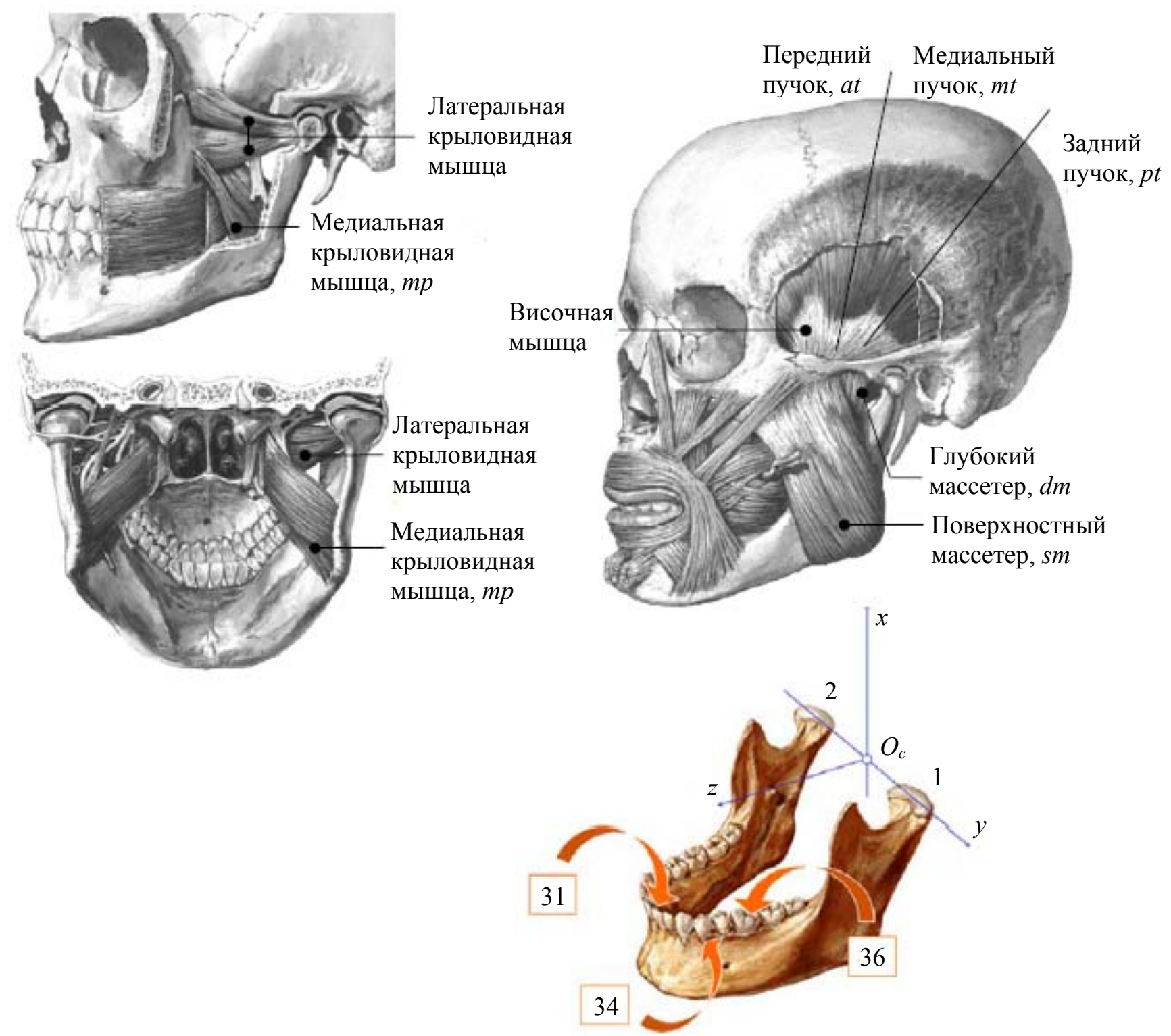

Рис. 1. Биомеханическая модель

\section{Мышицы-закрыватели челюсти}

Три пары мышц на левой и правой сторонах лица связаны в основном с нижней челюстью и принимают участие в переносе сил при разговоре или жевании: жевательные мышцы (masseters), средние крыловидные мышцы (medial pterygoid muscles) и височные мышцы (temporal muscles), см. рис. 1. Эти три пары мышц следует учитывать при построении математической модели сил в височно-нижнечелюстном суставе, чтобы получить данную точность результатов [18].

Жевательная мышца (masseter) - короткая и широкая, состоит из двух частей. Поверхностный массетер $(\mathrm{sm})$ - толстая мышца, проходящая от передней части скуловой дуги. Волокна этой мышцы вставляются в угол нижней челюсти и образуют мощный подъемник. Его направление образует пропульсию нижней челюсти. Глубокий массетер $(d m)$ меньше и проходит от задней части скуловой дуги. Его волокна вставляются в латеральную поверхность венечного отростка нижней челюсти и остаются почти перпендикулярными окклюзионной плоскости. 
Медиальная крыловидная мышца $(m p)$ остается параллельной внутренней стороне нижней челюсти. Она находится выше медиальной поверхности латеральной крыловидной плоскости и вставляется в заднюю и нижнюю части медиальной поверхности нижнечелюстной ветви. Эта мышца образует одновременно мощный подъемник и помогает в движении челюсти назад и вперед, а также из стороны в сторону.

Височная мышца начинается в височной ямке, проходит через скуловую дугу и присоединяется к нижнечелюстному венечному отростку. Эта мышца широкая и плоская и может быть разделена на три пучка, чтобы моделировать ее действие с помощью трех сил (см. рис. 1). Верхний пучок (at) одновременно толкает вперед и поднимает нижнюю челюсть. Медиальный пучок $(m t)$, волокна которого почти ортогональны окклюзионной плоскости, в основном поднимает челюстную кость. Наконец, волокна заднего пучка (pt), имеющего некоторые прикрепления к мениску височно-нижнечелюстного сустава, действуют в движении обратной пропульсии.

В этой части исследования мы будем считать, что сила тяжести и силы открывания челюсти не являются существенными и могут быть опущены в соответствии с работой [4].

\section{Волонтеры}

У взрослых людей жевательные мышцы, по-видимому, развивают силы, величины которых непосредственно связаны с их топологией. Эти величины меньше, чем их средние величины у гиперотклоняющихся от нормы людей и больше - для гипоотклоняющихся людей $[2,6,13]$. Такие корреляции между величинами и морфологиями могут говорить о том, что мышечная активность дает вклад в моделирование лицевого скелета или что мышечная активность меняется в соответствии с генетически заложенной формой лица.

Четыре волонтера со средними параметрами тела имели полный комплект зубов без зубных протезов или суставной патологии. Они не подвергались ортодонтическому лечению для исправления их суставных морфологических характеристик и мышечной физиологии. Протокол результатов был утвержден главой лаборатории и волонтерами студентами-медиками, давшими неформальное согласие и проявившими интерес к результатам.

\section{Статическое равновесие нижней челюсти}

При межзубном нагружении нижняя челюсть подвержена действию силы тяжести $\{W m\}$, мышц-открывателей челюсти $\{A o p / m\}$, мышц-закрывателей челюсти $\{A c l / m\}$, межзубной нагрузки $\{A s e / m\}$ и, наконец, двух суставов $\{A c m 1\}$ и $\{A c m 2\}$ (череп действует на левый и правый мыщелки нижней челюсти). С учетом этих шести торсоров условия статического равновесия нижней челюсти могут быть записаны в следующем виде:

$$
\{W m\}+\{\text { Aop } / m\}+\{\text { Acl } / m\}+\{\text { Ase } / m\}+\left\{\text { Acm }_{1}\right\}+\left\{\text { Acm }_{2}\right\}=\{0\} .
$$

Если не учитывать силу тяжести и силы мышц-открывателей челюсти, то глобальное действие $\{A c m\}$, передаваемое черепом на нижнюю челюсть, имеет вид

$$
\{A \mathrm{~cm}\}=\left\{\mathrm{Acm}_{1}\right\}+\left\{\mathrm{Acm}_{2}\right\}=-\{\text { Ase } / \mathrm{m}\}-\{\mathrm{Acl} / \mathrm{m}\} .
$$

Для оценки действия сил $\left\{A c m_{1}\right\}$ и $\left\{A c m_{2}\right\}$, передаваемых суставами, необходимо определить направления и величины силы межзубной нагрузки и величины усилий мышц-закрывателей нижней челюсти $\{A s e / m\}$ и $\{\mathrm{Acl} / \mathrm{m}\}$. 

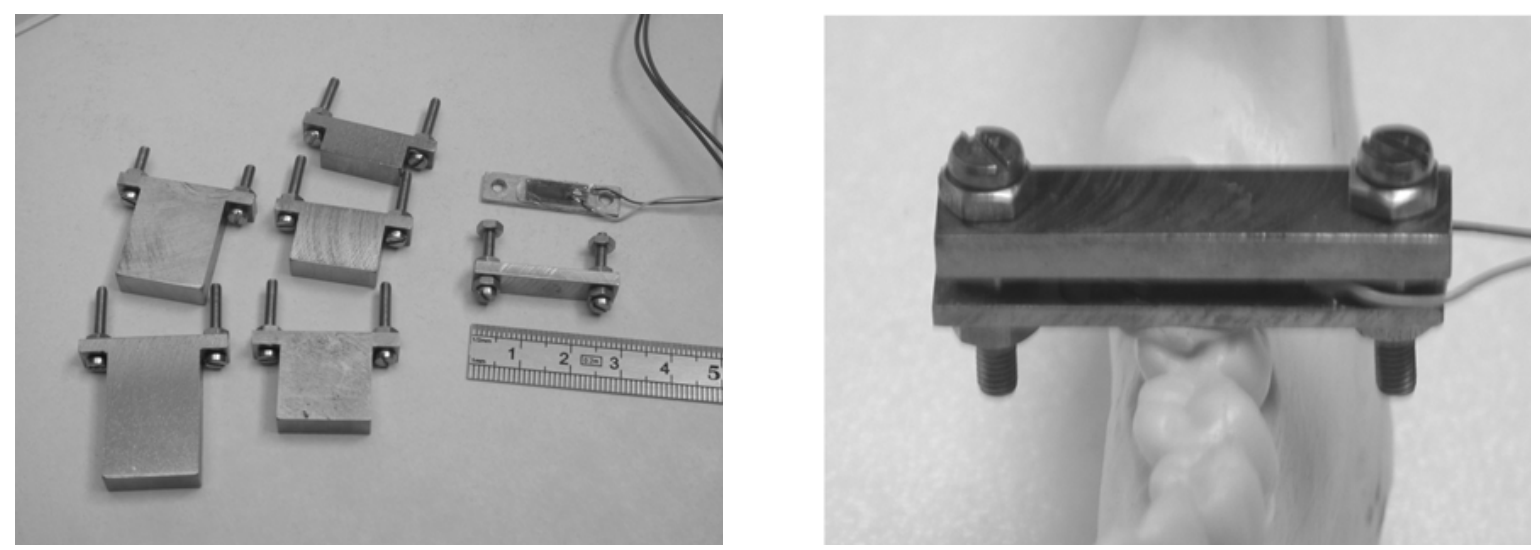

Рис. 2. Межзубный датчик

\section{Межзубная нагрузка}

Статическая сила кусания, приложенная к зубу нижней челюсти, была смоделирована и определена с помощью специально сконструированного датчика (рис. 2). Этот межзубный датчик также позволил нам управлять открыванием рта, используя две различные толщины (5 и 15 мм). In vivo это было успешно проделано между двумя резцами (31-21), двумя премолярами (34-24) и затем двумя молярами (36-26), что позволило определить $\{$ Ase $/ m\}$.

\section{контакта}

Направления сил мышц-закрывателей челюсти и расположение точек

Были сделаны четыре рассечения, чтобы исследовать контакты между нижнечелюстными мышцами и височной костью, а также рассмотрены прикрепления шести мышц-закрывателей челюсти. Фотографии и компьютерные изображения, полученные в лаборатории (используя Labview), были применены, чтобы экспериментально определить точки контакта (левая точка $C_{1}$ и правая точка $C_{2}$ ) и центры прикрепления мышц в морфологической системе координат Кампера (рис. 3). Вычисление направлений мышечных сил было выполнено на компьютере. Открытие рта, соответствующее двум толщинам (5 и 15 мм), было успешно изучено датчиком. Координаты, использованные для вычисления направлений суставных сил, соответствовали среднему из четырех рассечений.

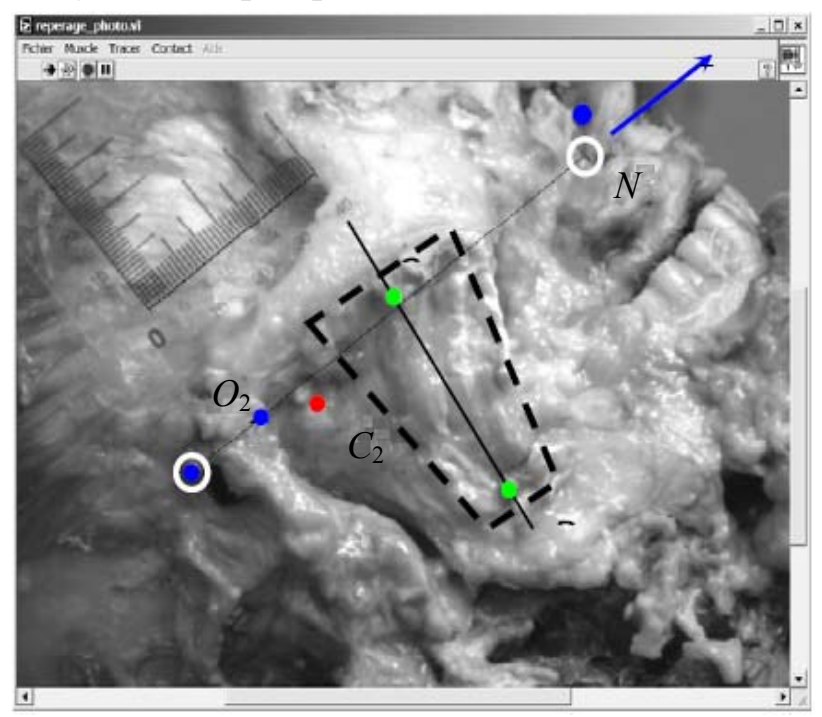

Рис. 3. Определение направлений мышечных сил (глубокий массетер) 


\section{Величины сил мышц-закрывателей челюсти}

In vivo данные по электромиографии и магнитно-резонансные изображения были использованы для измерения сил шести мышц-закрывателей челюсти [3].

Электромиографическая активность мылич

При значительных самопроизвольных сокращениях глобальная электромиограмма дает информацию об активности волокон мышц и величинах мышечных сил. Максимальная величина этих сил может быть достигнута, только если почти все волокна включены в процесс сокращения. Электрическая активность здесь выражается во внутримышечной биполярной форме. Так как края двух мышц не имеют какого-либо относительного перемещения, то длина мышцы остается постоянной и сокращение является изометрическим.

Расположение поверхностного массетера и височной мышцы определялось непосредственно. Глубокий массетер расположен в плотной анатомической зоне, а доступ к крыловидной мышце возможен только из ротовой полости. Для шести мышцзакрывателей были использованы внутримышечные нитевидные электроды. Две проволоки (платино-иридиевые, диаметр 20-30 мкм), изолированные нейлоновой оболочкой, были введены в гиподермическую иглу. Концы затем были обожжены, чтобы удалить изолятор. Два электрода затем были изогнуты, чтобы улучшить зацепление, когда игла была удалена.

Для использованного метода было необходимо строгое обоснование. Комната была освидетельствована DRASS (Direction Régionale des Affaires Sanitaires et Sociales) по рекомендации CCPPRB (Comité Consultatif pour la Protection des Personnes de Bordeaux). Имплантаты электродов были изготовлены доктором медицины.

При описанных изометрических условиях имеется линейное соотношение между сигналом электромиограммы и мышечной силой. Это соотношение часто используется в литературе $[8,15]$. Если сила, развиваемая мышцей, обозначается как $F$, то максимальное значение обозначается как $F_{\max }$. Аналогично $E M G r$ и $E M G r_{\max }$ обозначают текущее и максимальное среднеквадратичное значение (RMS) электромиографического сигнала $(E M G)$. Тогда, учитывая линейность, логично написать следующее соотношение:

$$
F_{\text {muscle }}=\left(F_{\text {muscle } \max } / E M G r_{\text {max }}\right) E M G r,
$$

где

$$
E M G_{R M S}=\left(1 / T \int_{t-T / 2}^{t+T / 2}(E M G)^{2} d t\right)^{1 / 2}, \mathrm{MB} .
$$

При самопроизвольном сокращении, когда почти все волокна мышц вовлечены (максимальная сила кусания, создаваемая волонтером), в равной степени линейное соотношение существует между максимальной мышечной силой и ее главным физиологическим сечением в состоянии покоя. Если площадь этого сечения обозначить как $S_{\max }$, а $K$ есть постоянный коэффициент, который характеризует группу мышц, то соотношение может быть записано в следующем виде:

$$
F_{\text {muscle } \max }=S_{\max } \cdot K .
$$

В зависимости от морфологии мышц коэффициент $K$ равен от 0,6 до $0,9 \mathrm{H} / \mathrm{m}^{2}$. Эти данные взяты из литературы $[13,14,16]$, а текущая мышечная сила выражается в виде

$$
F_{\text {muscle }}=\left(S_{\max } \cdot K / E M G r_{\max }\right) E M G r .
$$



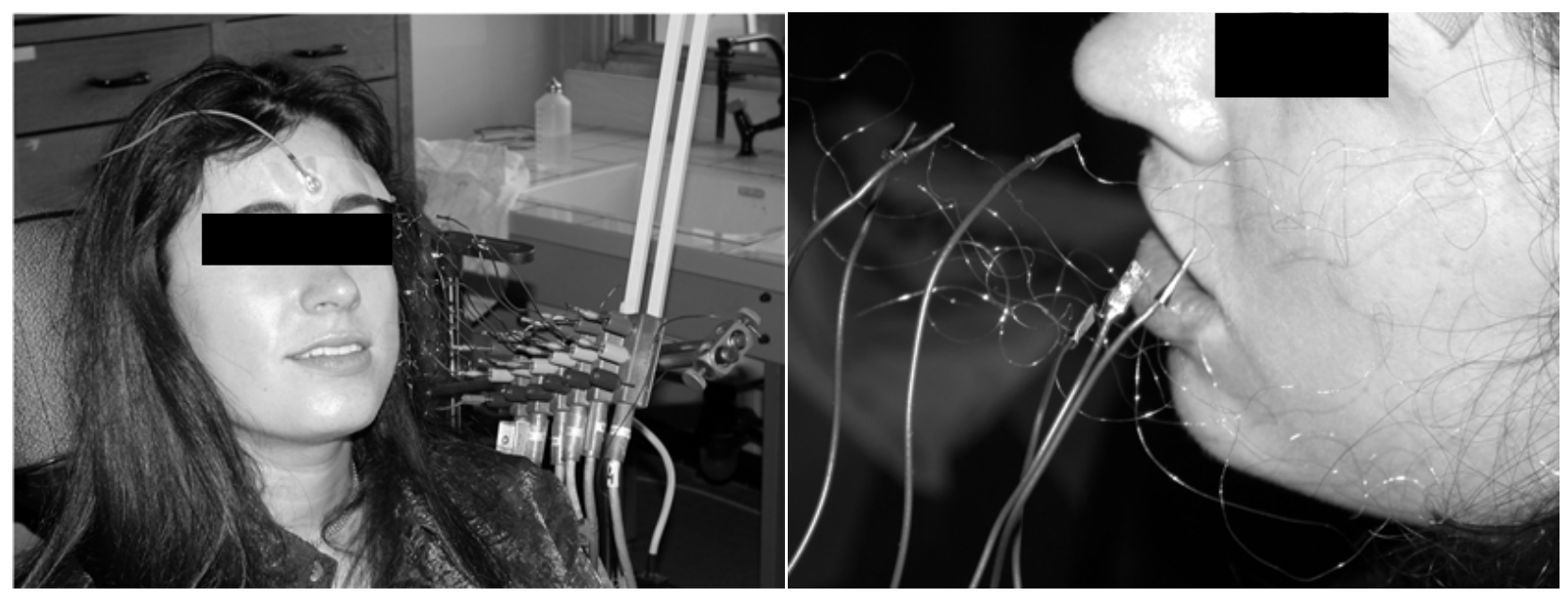

Рис. 4. Определение электромиографических мышечных сигналов

Чтобы использовать это соотношение для каждой мышцы-закрывателя челюсти, необходимо определить $E M G r_{\max }$, повторяя самопроизвольное изометрическое максимальное сокращение, и $S_{\max }$, начиная с проверки магнитно-резонансного изображения.

При регистрации электромиографических сигналов прибор регистрирует несколько последовательных функций:

- обработка сигнала с использованием нитевидных электродов (рис. 4), усиление и фильтрация;

- регистрация данных и компьютерная обработка.

Было принято предположение о морфологической симметрии лица, чтобы уменьшить сильный дискомфорт, испытываемый волонтером. Следовательно, области сечений мышц были изучены только на левой стороне лица. Электроды были равным образом распределены на левой стороне лица, и датчик был расположен последовательно между резцами (31/21), первыми премолярами (34/24) и первыми молярами (36/26).

C учетом идеальной геометрической симметрии лица ситуация (31/21) соответствовала случаю плоского нагружения. С другой стороны, для несимметричного нагружения, например между премолярами, датчик сначала был расположен на зубе 34. Электроды и датчик сначала находились на том же профиле, и датчик сдвинулся к зубу 44. Электроды и датчик были поэтому расположены на различных профилях. С использованием этого метода электромиографические сигналы двух профилей (ipsi and contro) могут быть рассчитаны, избегая при этом билатеральную имплантацию электродов.

Физиологические сечения мыши

Магнитно-резонансное изображение дает образы с высокой степенью точности. Для определения главного сечения мышцы нужно знать главное направление (или положения центров прикрепления). Для этого необходимо сделать несколько параллельных и близких измерений, ортогональных по отношению к этой продольной оси мышцы.

Компьютерные изображения анализируются с помощью программ обработки изображений (Osiris, Hopitaux Universitaires de Genève, Switzerland). Модуль позволяет выбрать одну область изображения и охарактеризовать ее. Например, интересующая нас область позволяет определить границы двух массетеров (рис. 5). Программа обнаружила, что полная область сечения равна примерно $454 \mathrm{mм}^{2}$. 


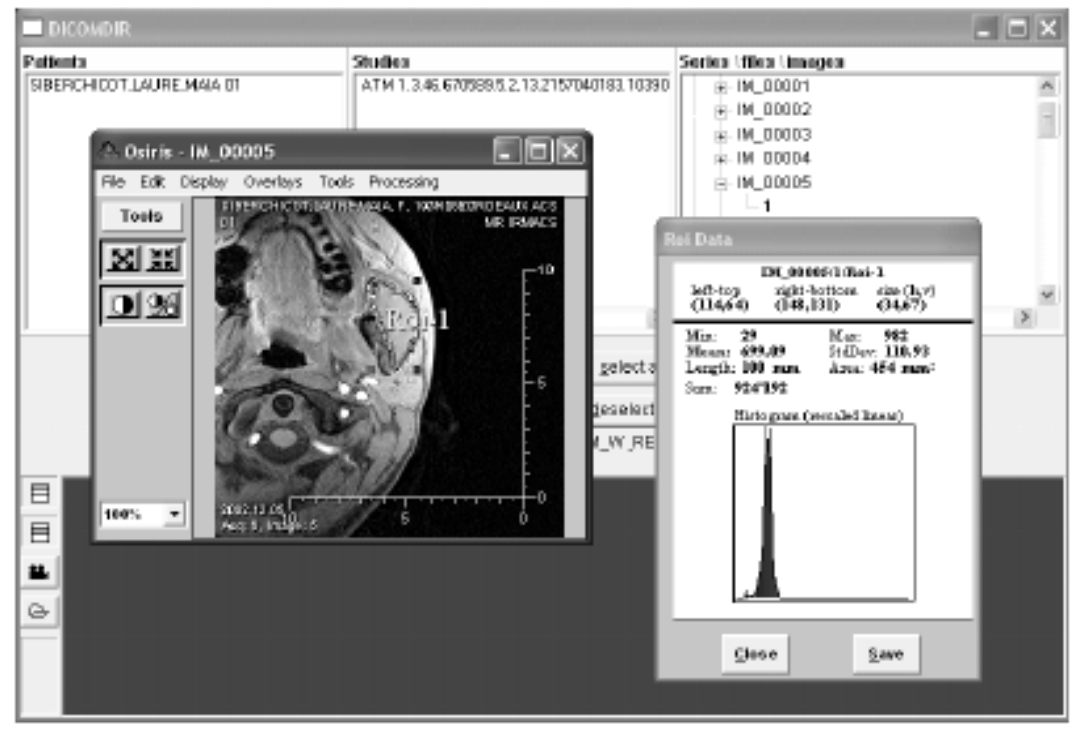

Рис. 5. Определение сечений мышцы

\section{Получение и решение системы уравнений}

С помощью базиса $b$ усилия, воспринимаемые левым суставом, могут быть представлены в виде

$$
\left\{A c m_{1}\right\}=\left\{X_{1} \mathbf{x}+Y_{1} \mathbf{y}+Z_{1} \mathbf{z} ; L_{1} \mathbf{x}+M_{1} \mathbf{y}+N_{1} \mathbf{z}\right\}
$$

в точке $C_{1}$, где сила имеет вид

$$
X_{1} \mathbf{x}+Y_{1} \mathbf{y}+Z_{1} \mathbf{z}
$$

Момент в точке $C_{1}$ представляется в виде

$$
L_{1} \mathbf{x}+M_{1} \mathbf{y}+N_{1} \mathbf{z} .
$$

Записав уравнения равновесия нижней челюсти в точке $C_{2}$ (de Leva, 2008 [7]), этот момент можно представить в виде

$$
L_{1} \mathbf{x}+M_{1} \mathbf{y}+N_{1} \mathbf{z}+C_{2} C_{1} \times\left(X_{1} \mathbf{x}+Y_{1} \mathbf{y}+Z_{1} \mathbf{z}\right)
$$

в точке $C_{2}$.

В базисе $b$ усилия, воспринимаемые правым суставом, логично записать в виде

$$
\left\{A c m_{2}\right\}=\left\{X_{2} \mathbf{x}+Y_{2} \mathbf{y}+Z_{2} \mathbf{z} ; L_{2} \mathbf{x}+M_{2} \mathbf{y}+N_{2} \mathbf{z}\right\}
$$

в точке $C_{2}$.

Не делая никаких гипотез, отметим, что глобальное воздействие $\{A c m\}$ имеет 12 неизвестных компонент:

$$
X_{1}, Y_{1}, Z_{1}, L_{1}, M_{1}, N_{1}, X_{2}, Y_{2}, Z_{2}, L_{2}, M_{2}, N_{2} \text {. }
$$

Таким же образом воздействие на нижнюю челюсть со стороны мышцы с номером $i$ имеет вид

$$
\{\text { A muscle } i / m\}=\left\{\mathbf{F}_{i}=F_{i} \mathbf{u}_{i} ; \mathbf{O}\right\}
$$

в точке $B$ (см. рис. 3), центре прикрепления мышцы с номером $i$. 
Уравнение аналогично для всех 12 мышц. Величина $i$ меняется от 1 до 12. Для каждой мышцы алгебраическое усилие $F_{i}$ вычисляется исходя из электромиографических данных. Направления единичных векторов $\mathbf{u}_{i}$, соответствующих последовательным двум открытиям (5 или 15 мм) и трем положениям датчика, были определены путем рассечений (см. рис. 3). Компоненты $\mathbf{u}_{i}$ и, следовательно, компоненты $\mathbf{F}_{i}$ в базисе $b$ тогда могут быть определены. Глобальные воздействия мышц-закрывателей челюсти комбинируют 12 известных сил:

$$
\{A c l / m\}=\sum\left\{A_{\text {muscle }} i / m\right\}
$$

для $i$ от 1 до 12 .

Наконец, воздействие, соответствующее нагрузке $F$, зарегистрированное сенсором, равно

$$
\{\text { Ase } / m\}=\{\mathbf{F}=F \mathbf{u} ; \mathbf{O}\}
$$

в точке $T$, соответственно зубу 31,34 или 36 (рис. 1).

Единственный вектор и был вычислен по нормали к плоскости биссектрисы, соответствующей открытию рта. Компоненты и и, следовательно, компоненты $\mathbf{F}$ в базисе $b$ могут затем быть вычислены.

Гиперстатическая система (1), выражающая статическое равновесие нижней челюсти, была записана в точке $C_{2}$. Уравнения включали 12 неизвестных компонент. Если законы, которые характеризуют поведение сустава (число степеней свободы), неизвестны, то остается невозможным вычислить силы, передаваемые при нагружении через суставные контакты. Оценка этих воздействий налагает реалистические допущения, которые должны быть подтверждены.

Височно-нижнечелюстные суставы являются сложными суставами. Каждый сустав включает мениск и капсулу, окружающую две суставные поверхности. Присутствие смазывающей синовиальной жидкости внутри этой синовиальной капсулы делает движения качения и скольжения гораздо более легкими. Это наблюдение позволяет предположить, что четыре компоненты $M_{1}, N_{1}, M_{2}, N_{2}$ остаются близкими к нулю.

Так как мышечные силы и нагрузка известны, то две компоненты $L_{1}$ и $L_{2}$ включают действие трения, воздействия сухожилия и мышц, которые не были учтены вокруг оси $\left(C_{2}, \mathbf{x}\right)$. Уравнение моментов вокруг оси $\left(C_{2}, \mathbf{x}\right)$ должно быть проверено; решение системы затем используется при численной оптимизации, минимизируя данный критерий. Выбранный критерий состоял в минимизации суммы $\left(L_{1}+L_{2}\right)$.

Шесть компонент сил $X_{1}, Y_{1}, Z_{1}, X_{2}, Y_{2}$ и $Z_{2}$ остаются неизвестными. Начиная с рассечений и геометрического анализа, две геометрические модели височнонижнечелюстного сустава были построены (рис. 6).

Первая модель $(S M)$ соответствовала симметричному нагружению с датчиком в положении 31 (рис. $6, a$ ). Для каждого мыщелка она включала одну точку контакта или «пару - шар и плоскость» (1BPP, сустав из двух звеньев, состоящих из шара и плоскости). Вторая модель (DSM) соответствовала несимметричному нагружению с датчиком в положении 34 или 36 (рис. 6, б). Эта модель представляла одну точку контакта для левого мыщелка нижней челюсти или «пару - шар и плоскость» $(1 B P P)$ и «сферическую пару» (1SP, сустав из двух звеньев, позволяющих сферическое движение одного звена относительно другого звена) для правого мыщелка. 


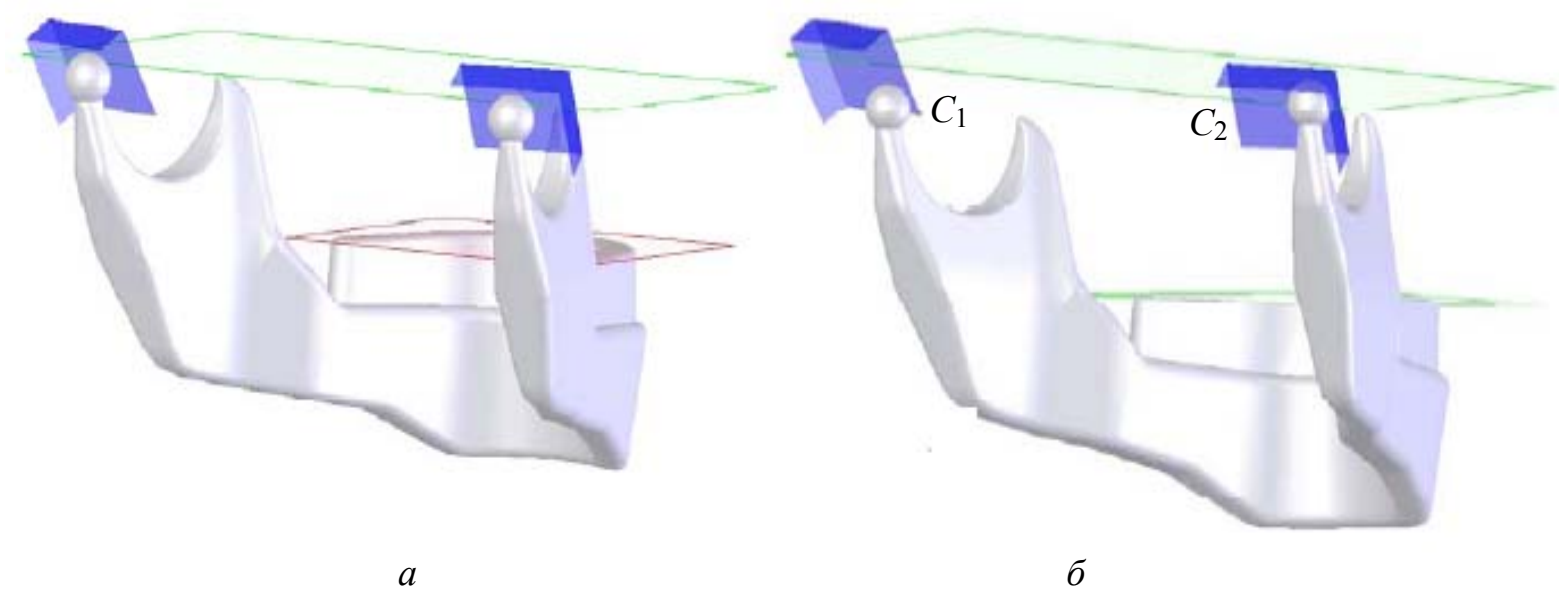

Рис. 6. Геометрические модели:

$a-S M, 1 B P P$ и $1 B P P ; \sigma-D S M, 1 B P P$ (слева) и $1 S P$ (справа)

Для оценки компонент сил, передаваемых при суставных контактах, решение системы уравнений было проведено на компьютере. Для симметричного нагружения (случай $S M$ ) компоненты $X_{1}$ и $X_{2}$ остаются неопределенными. Два мыщелка подвержены равным сагиттальным компонентам. Для случая DSM (несимметричного нагружения), если игнорировать трение, компонента $X_{2}$ остается неопределенной, а компонента $X_{1}$ исчезает.

\section{РезУльтаты}

В этом разделе представляются и обсуждаются результаты, полученные при определении мышечных и суставных сил.

\section{Силы мышц-закрывателей челюсти}

Силы, развиваемые мышцами в экспериментальных ситуациях, не могут быть сравнены, если датчик не показывает ту же нагрузку для всех тестируемых ситуаций. Чтобы обойти эту трудность, мышечные силы $F_{i}$ были преобразованы в $F_{i}$ unit для межзубной силы, которая всегда равна $1 \mathrm{H}$. Если $F$ представляла силу, регистрируемую датчиком в текущем тесте, то преобразование было следующим:

$$
F_{i \text { unit }}=F_{i} /|F| \text {. }
$$

Табл. 1 показывает величины сил для шести пар мышц-закрывателей челюсти. В каждой позиции три теста проведены для каждого из четырех волонтеров. Средние значения были вычислены на основе 12 значений.

Вклад задней височной мышцы остается слабым в сравнении с вкладом двух других пучков мышц (медиальной и передней): деление височной мышцы на два пучка кажется достаточным. Цель заключалась в том, чтобы улучшить точность результатов и обойти трудность векторизации этой мышцы, которая выглядит как веер. Из-за волос волонтеров поверхностные электроды могут быть использованы только для переднего пучка височной мышцы. Подавление четырех нитевидных электродов представляло интерес и уменьшило неприятные ощущения волонтеров. 


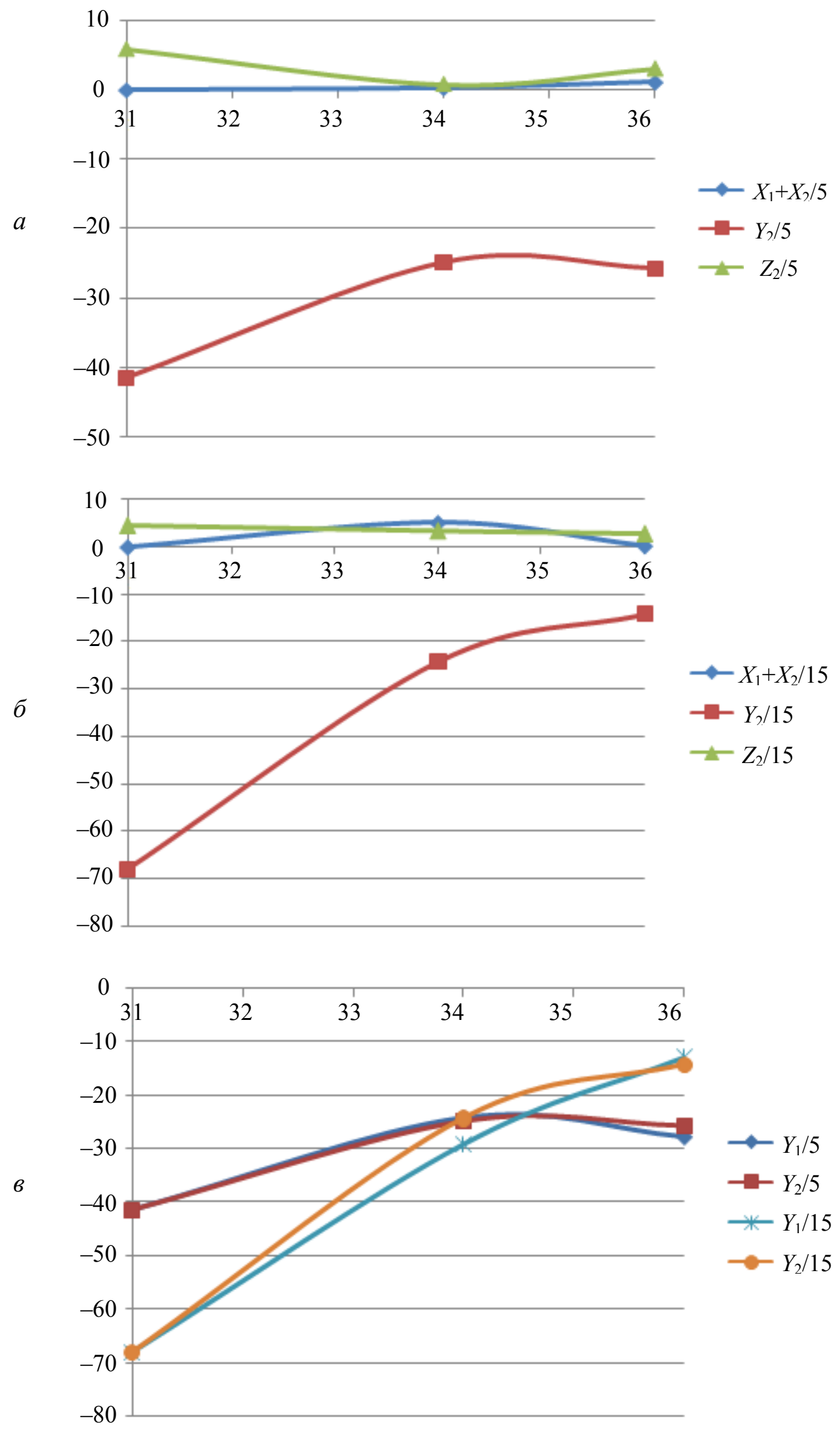

Рис. 7. Суставные компоненты $(\mathrm{H})$ при нагружении силой $1 \mathrm{H}$ последовательно на зуб 31,34 и $36: a$ - тонкий датчик (5 мм), $\sigma$ - широкий датчик (15 мм), в - сравнение левого и правого датчиков (5 и 15 мм) 
Таблииа 1

Силы мышц-закрывателей челюстей (Н), соответствующие нагрузке в 1 Н на зуб

\begin{tabular}{|c|c|c|c|c|c|c|c|}
\hline \multirow{2}{*}{ Зуб } & \multirow{2}{*}{ Датчик } & \multicolumn{6}{|c|}{ Мышца } \\
\cline { 3 - 8 } & & $d m$ & $s m$ & $m p$ & $a t$ & $m t$ & $p t$ \\
\hline & & слева/справа & слева/справа & слева/справа & слева/справа & слева/справа & слева/справа \\
\hline 31 & 5 & $4,72 / 4,72$ & $18,72 / 18,72$ & $20,78 / 20,78$ & $1,07 / 1,07$ & $0,51 / 0,51$ & $0,35 / 0,35$ \\
\hline 34 & 5 & $3,65 / 4,65$ & $8,16 / 11,87$ & $8,62 / 7,05$ & $5,07 / 1,73$ & $2,71 / 0,52$ & $0,65 / 0,33$ \\
\hline 36 & 5 & $2,99 / 3,78$ & $7,26 / 7,32$ & $13,59 / 10,41$ & $6,32 / 4,51$ & $3,13 / 1,70$ & $0,03 / 0,18$ \\
\hline 31 & 15 & $6,02 / 6,02$ & $21,73 / 21,73$ & $42,16 / 42,16$ & $3,70 / 3,70$ & $2,59 / 2,59$ & $0,43 / 0,43$ \\
\hline 34 & 15 & $3,53 / 3,54$ & $5,89 / 7,72$ & $20,36 / 8,76$ & $4,72 / 2,47$ & $2,19 / 1,04$ & $0,77 / 0,64$ \\
\hline 36 & 15 & $1,67 / 2,80$ & $3,75 / 4,90$ & $4,86 / 3,68$ & $3,29 / 2,71$ & $1,50 / 1,63$ & $1,02 / 0,17$ \\
\hline
\end{tabular}

\section{Симметричное нагружение, SM-модель}

Численные значения суставных сил соответствуют единичной силе, регистрируемой датчиком (табл. 2). Рис. 7 описывает изменения в сагиттальных компонентах для каждого датчика: для самого тонкого датчика (5 мм) на рис. 7, $a$, для самого толстого датчика (15 мм) на рис. 7,6 . На рис. 7, в сравниваются левые и правые y-компоненты для двух датчиков.

Два нижнечелюстных мыщелка имеют одинаковые сагиттальные компоненты при симметричном нагружении. Для открытия рта, соответствующего самому широкому датчику (15 мм), суставные точки контакта расположены около височных мыщелков. Компоненты $Y_{1}$ и $Y_{2}$ тогда достигают своих максимальных абсолютных значений (см. рис. 7, б). Ветвь нижнечелюстного мыщелка, направленная по оси $y$ при открытии челюстей, тогда подвержена квазичистому сжатию. Две компоненты $Z_{1}$ и $Z_{2}$ остаются малыми, и два отношения $Z_{1} / Y_{1}$ и $Z_{2} / Y_{2}$ остаются менее $15 \%$.

\section{Несимметричное нагружение, DSM-модель}

В табл. 2 представлены алгебраические величины суставных компонент, если нагрузка последовательно прикладывается к резцу, премоляру и моляру. Для открытия рта, соответствующего самому тонкому датчику (5 мм), значения $Y_{1}$ и $Y_{2}$ остаются одинаковыми (рис. 7, a). Для позиций от 31 до 36 абсолютные значения $Y_{1}$ и $Y_{2}$ значительно уменьшаются. Компонента $Z_{1}$ остается очень малой и почти постоянной, в то время как $Z_{2}$ стремится к нулю. От позиции 31 к позиции 36 абсолютные значения $Y_{1}$ и $Y_{2}$ также уменьшаются при использовании самого широкого датчика (см. рис. 7, б). Кроме одной точки два отношения $Z_{1} / Y_{1}$ и $Z_{2} / Y_{2}$ остаются менее $20 \%$.

Силы в суставах (Н), соответствующие нагрузке 1 Н на зуб

Таблииа 2

\begin{tabular}{|c|c|c|c|c|c|c|c|c|}
\hline \multirow{2}{*}{ Зуб } & \multirow{2}{*}{ Датчик } & \multicolumn{9}{|c|}{ Компонента } & \multicolumn{2}{c|}{ Отношение } \\
\cline { 3 - 9 } & $X_{1}+X_{2}$ & $Y_{1}$ & $Y_{2}$ & $Z_{1}$ & $Z_{2}$ & $Z_{1} / Y_{1}$ & $Z_{2} / Y_{2}$ \\
\hline 31 & 5 & 0 & $-41,4$ & $-41,1$ & 6,0 & 6,0 & $-0,14$ & $-0,14$ \\
\hline 34 & 5 & 0,3 & $-24,1$ & $-24,9$ & 4,2 & 0,9 & $-0,17$ & $-0,03$ \\
\hline 36 & 5 & 1,2 & $-27,7$ & $-25,7$ & 4,7 & 3,1 & $-0,17$ & $-0,12$ \\
\hline 31 & 15 & 0 & $-68,0$ & $-68,0$ & 4,5 & 4,5 & $-0,07$ & $-0,07$ \\
\hline 34 & 15 & 5,1 & $-29,1$ & $-24,3$ & 5,1 & 3,4 & $-0,18$ & $-0,14$ \\
\hline 36 & 15 & 0,3 & $-12,8$ & $-14,3$ & 4,1 & 2,8 & $-0,32$ & $-0,20$ \\
\hline
\end{tabular}




\section{ОБСУЖДЕНИЕ}

\section{Изменения в протоколе}

Знание о направлении мышечных сил, необходимых для равновесия нижней челюсти, можно получить in vivo. Различие персональной мышечной $3 D$-модели должно быть получено с использованием диффузионного магнитно-резонансного метода, который позволяет получить изображения in vivo биологических тканей, взвешенных с локальными характеристиками диффузии воды. Интенсивность изображения уменьшается в зависимости от анизотропной микроструктуры мышцы, в которой имеется диффузия молекул воды.

Как было показано, можно облегчить обеспечение электродов и этим упростить протокол. Уменьшение неприятных ощущений волонтеров, вероятно, также позволит улучшить точность результатов.

Направления сил, вызываемых массетером и медиальными крыловидными мышцами, остаются почти параллельными, и поэтому можно учитывать замену крыловидного электрода в полости рта, назначая скорректированное сечение массетера.

\section{Точность первых результатов}

В данной работе рассмотрены шесть мышц-закрывателей челюсти, в то время как мышцы-открыватели не рассмотрены. Так как мышца действует в синергии с несколькими другими мышцами, то результаты, описанные в данной статье, не нужно считать конечными, они в действительности открывают возможность рассматривать мышцы-открыватели челюсти. Следующий шаг - изучить относительный вклад мышцоткрывателей и мышц-закрывателей челюсти и проверить гипотезу, сформулированную Caix [4]. Мы также улучшим численные результаты, используя большую модель популяции [5].

\section{БЛАГОДАРНОСТИ}

Авторы благодарны фондам $C N R S, R F B R$ и $M A E$. Также авторы благодарят волонтеров за их терпение.

\section{СПИСОК ЛИТЕРАТУРЫ}

1. Baird D.N., Rea J. The temporomandibular joint implant controversy: a review of autogenous alloplastic materials and their complications // Journal of Nutritional and Environmental Medicine. - 1998. - Vol. 8, № 3. - P. 289-300. DOI: http://dx.doi.org/10.1080/13590849862078

2. Belguedj M. L'organisation du développement vertical des structures cranio-facio-cervicales, Laboratoire d'odontologie, Université du Droit et de la Santé. - Lille, 2000.

3. Bouisset S., Maton B. Muscles, posture et movement. - Paris: Hermann, 1995.

4. Caix P., Carles J. Anatomie fonctionnelle / Laboratoire d'Anatomie Médico-Fonctionnelle et Chirurgicale, Université Bordeaux 2. - Bordeaux, 2000.

5. Coutant J.C., Mesnard M., Morlier J., Ballu A., Cid M. Discrimination of objective kinematic characters in temporomandibular joint // Archives of Oral Biology. - 2007. - Vol. 53, № 5. - P. 453-461. DOI: http://dx.doi.org/10.1016/j.archoralbio.2007.11.010

6. Darqué J., Darqué F., Boileau M.J. Dimension verticale // Congrès de la Société Française d'Orthopédie Dento-Faciale. - Bordeaux, 1989.

7. de Leva P. Anticrossproducts and cross divisions // Journal of Biomechanics. - 2008. - Vol. 41. - P. 17901800. DOI: http://dx.doi.org/10.1016/j.jbiomech.2007.09.030

8. El-Bilay T.H., Abdelhameed M. Quantitative calculations of temporomandibular joint reaction forces as a function of mandibular lengthening // The TMJournal. - 2004. - Vol. 3, № 7. - P. 1-11.

9. Karray F., Fakhfakh Z., Kallel A., Turki R. Interface tissus vivants-matériaux inertes // Canadian Journal of Physics. - 1999. - Vol. 77. - P. 745-750. DOI: http://dx.doi.org/10.1139/p99-032 
10. Koolstra J.H., van Eijden T.J., Weijs W.A., Naeje M.A. A 3D mathematical model of the human masticatory system predicting maximum possible bite force // Journal of Biomechanics. - 1988. - Vol. 21. P. 563-573. DOI: http://dx.doi.org/10.1016/0021-9290(88)90219-9

11. Layrolle P. Ostéointégration d'implants orthopédiques et dentaires // Matériaux et Techniques. - 2006. Vol. 94. - P. 71-76. DOI: http://dx.doi.org/10.1051/mattech:2006027

12. Mesnard M. Elaboration et validation d'un protocole de caractérisation de l'articulation temporomandibulaire: Thèse de doctorat de l'Université de Bordeaux, no. 3130. - Bordeaux, 2005.

13. Moller E. The chewing apparatus // Acta Physiologica Scandinavia. - Copenhagen, 1966.

14. Nahmias I. Contribution à l'étude de la cinématique mandibulaire // Orthodontie / Université Paris 5. - Paris, 2000.

15. Pruim G.J., De Jongh H.J., Ten Bosch J.J. Forces acting on the mandible during bilateral static bite at different bite force levels // Journal of Biomechanics. - 1980. - Vol. 13. - P. 755-763. DOI: http://dx.doi.org/10.1016/0021-9290(80)90237-7

16. Pruim G.J., Ten Bosch J.J., De Jongh H.J. Jaw muscle EMG-activity and static loading of the mandible // Journal of Biomechanics. - 1978. - Vol. 11. - P. 389-395. DOI: http://dx.doi.org/10.1016/00219290(78)90073-8

17. Speculand B., Hensher R., Powell D. Total prosthetic replacement of the TMJ: experience with two systems 1988-1997 // British Journal of Oral and Maxillofacial Surgery. - 2000. - Vol. 38, № 4. - P. 360-369.

18. Throckmorton G.S. Quantitative calculation of the temporomandibular joint reaction forces // Journal of Biomechanics. - 1985. - Vol. 18. - P. 445-452. DOI: http://dx.doi.org/10.1016/0021-9290(85)90279-9

19. Wolford L.M., Dingwerth D.J., Talwar R.M., Pitta M.C. Comparison of two temporomandibular joint prosthesis systems // Journal of Oral and Maxillofacial Surgery. - 2003. - Vol. 61, № 6. - P. 685-690. DOI: http://dx.doi.org/10.1053/joms.2003.50112

\title{
CHARACTERIZATION OF THE MUSCULAR AND ARTICULAR FORCES EXERTED ON THE MANDIBLE. EXPERIMENTAL AND CALCULATIVE METHODS
}

\author{
M. Mesnard, A. Mesnard (Bordeaux, France), A. Ramos (Aveiro, Portugal), \\ V.A. Lokhov, Y.I. Nyashin (Perm, Russia)
}

This paper presents a 3D method of quantifying and analyzing the muscle forces and joint forces exerted on the mandible under loadings. The experimental protocol, the hypotheses, the writing and then the resolution of the equations system that is related to the static equilibrium of the mandible, are described and applied using data from four volunteers. A sensor was designed to simulate and register the bite force between two teeth, and was applied successively between two incisors, two premolars, and then two molars. Dissections were carried out to determine the contacts between the mandible condyles and the temporal bone and also the insertions of the jaw-closing muscles. The directions of the muscular forces were thus determined in a morphological coordinate system. Magnetoresonance imaging and electromyography data were used in vivo to evaluate the magnitudes of six muscle forces on each side of the face. The resolution of the equation system written to calculate the forces transmitted through the articular contacts was computer-assisted. For an identical loading applied successively to positions 31,34 and 36 , on the basis of twelve recordings, the results show that the sagittal components of the articular forces are strongly influenced by interdental distance. In position 31, the vertical component is thus multiplied by 1.65 while the interincisor distance increases from 5 to 15 millimeters. The magnitudes of the articular forces are strongly influenced by modifications of the mouth aperture and by the directions of the resulting muscular forces.

Key words: biomechanical characterization, muscle forces, joint forces, mechanical modelling, temporomandibular joint implant. 